\title{
Managing and Reducing Handoffs Latency in Wireless Local Area Networks using Multi-Channel Virtual Access Points
}

\author{
Kamran Javed ${ }^{1}$, Fowad Talib ${ }^{2}$, Mubeen Iqbal ${ }^{3}$, Asif Hussain Khan ${ }^{4}$ \\ Faculty of Computing and Information Technology \\ University of Sialkot, Sialkot, Pakistan
}

\begin{abstract}
The time is era of computer technology and relevant hybrid disciplines to emerge as a multi impact entity in the technological world. In the same stream where user of technology is increasing the expectation from the technology are also expedite, thus users need to have high speed network to support high speed devices, especially in the technical world where palm computers hit the market at its excel. High data transfer rate should be enough supportive to the environment of next generation wireless networks. Mobility is another added factor to high speed connectivity issues. Users for enormous application would like to use a network which is heterogeneous in nature, such as high availability and high bandwidth to avoid issues in real time applications, video streaming and including VoIP and multi-media over the mobile networks. Thus mobile communication access massively relies on the continuous network availability which is done through handoffs, ensures the seamless transfer of device from one AP to another. In this paper, I have extracted the novel approach of multi-channel virtual access points which will nullify or reduce the handoffs latency.
\end{abstract}

Keywords-Technology; mobility; heterogeneous; bandwidth; video streaming; mobile network; VoIP; handoffs

\section{INTRODUCTION}

Mobility of the nodes is obsessive compulsion of today's wireless networking system. Mobility requires the mechanism of handoffs for the continuous connectivity. During handoffs the mobile nodes change their connection from one AP to the other and this actually changes the channel of the communication with that particular AP. Changing the channel means that altering the frequency, time slot and code spreading, or combination of them as in TDMA, FDMA, CDMA and hybrid schemes. Changing the channel during the use of multi-media, VoIP, streaming, data or voice calls interruption is experienced or may be in some cases crashing the ongoing communication. The mobile nodes can get connected to any wireless network subject to the availability such as GPRS or WI-Max. These networks requires high data rate to provide good services to mobile nodes, for this purpose a handoff technique called vertical handoff is required to switch between the networks seamlessly, but the focus of this study is handoff between APs rather than inter-network transition [1].

The focus of the study requires the certain parameters to be fulfilled for the seamless switching between the APs for easy configuration, cheap hardware and for the sake of rapid deployment in the existing system. IEEE 802.11 standards of wireless networks can do the best job for the purpose taking in account the above mentioned requirements. It is well established and well learned fact that the APs transport traffic between the clients (or nodes) and network infrastructure (which is mostly based on the wired network) to establish communication sessions between the nodes.

When a station (in this paper "station" refers to nodes on the move) moves away from an AP, after attaining certain distance, the signal strength drops below the threshold level of the connectivity, the station immediately starts searching for the next nearest AP to sustain its connectivity, unless found, its transceivers stops working means nor sending neither receiving anything, this "blackout" of signals impact the communication going on and multi-media applications. Threshold of the signal strength and finding the new AP's signal majorly depends upon the service range of the AP. Many solutions have been proposed till now for avoiding this problem. Vertical handoffs, as mentioned earlier and the Horizontal handoffs techniques are the alike but differentiated based on the network type being used while transition from one AP to another. Virtual access point is another approach to handle and overcome the issue in wireless networking and is focus of this study. (Grunenberger and Rousseau, 2010). Virtual access points with multi channels will support the purpose of this study, i.e. to provide seamless handoff in wireless networking. Added advantages are increased network capacity and interference avoidance along with its main feature that is handing off station from one AP to another without network traffic disruption. To support network traffic exchange between nodes through multi channels virtual access points a Distributed system protocol (inter-AP) will be helpful to achieve the purpose. Inter-AP protocol specifically in WLANs is supportive in changing the channels while transmitting packets and without interrupting the communication between station and AP.

\section{EASE OF USE HANDOFFS PROCESS IN IEEE 802.11 AND RELATED WORK}

If the handoffs delay is significant or longer than some milliseconds, it certainly will introduce a disordered behavior and hence will bring up sizeable and considerable disruption in the network traffic. According to (ITU,2003) if the handoff delay excels 150 millisecond it will not be able to provide good quality services or specifically good voice quality in case of 
VoIP, as some times searching a new AP in the neighboring location, finding the channel of that AP ready to associate itself with the station can take up to 2 seconds. The handoffs procedure of a station can be depicted in two points.

Letting client search for the new AP available to get itself associated with. Station searching for the AP will send a probe request to locate the vacant channel operating on the AP in range.

On successful acknowledgment the AP then associated with the station through the determination of re-authentication process of association of the new AP for the station.

In this case the MAC handoff is initiated by the station. The above handoffs procedure is based on the Shin et al 2004, which proposed the reduction of latency in handoffs at the MAC layer of OSI Model. In terms of delay there are three components of the MAC layer as probe, authentication \& association.

A scheme was proposed for the handoffs through selecting and caching mechanism is focused on the behavior of the station, because in this case the handoffs process is controlled by the mobile station. However proposed method helps in reduce the latency and ascertained that the seamless communication can be achieved in wireless networks. The probing process or channel scanning is the time taken by the AP can be proactive or selective scanning eavesdropping and can be reduced for the authentication information through proactive distribution. (Wu et al, 2007) (Liao and Gao, 2006).

Channels with less network traffic, not overlapped, increased network capacity, reduced interference and latency can be used for the communication between the APs and thus can listen to the neighboring APs. So the virtual access point concept can be used to lever handoffs in multichannel WLANs.

\section{VAP CONCEPT}

Virtual access point is underneath part of the actual access point and can be thought as an extensible access point as it seems different AP to the node because it ought to be with different identity i.e. SSID that is because the VAP works at the MAC level to replicate the functioning of the real Access point. The real AP can transmit as much VAPs as required, i.e. broadcasting different SSIDs from same physical AP, but here as an example an AP is broadcasting 3 SSIDs (VAPs) this means that information of nodes can be transmitted to 3 other real APs, that is why it can transfer the control visitors of three separate APs, one for the each separate VAP it facilitates. [2].

Virtual access point imitates and behaves like a physical access point. Based on the per radio system a private or dedicated entry way is designed. As already discussed, we can achieve the hand off by introducing two stereos as gadgets of APs to reduce the latency, where one stereo will keep verifying the next and new AP while keeping the connection between node and BSSID on the other stereo. Each stereo can have 16 virtual access points, each with unique entry way ranging from 0 to 15 . However only one entry way is allowed according to standards and that is VAP 0 . As we know that each entry way is uniquely associated to premeditated user configured service set identifier and basic service set identifier. Apart from VAP 0 the VAP $1-15$ is virtual access Factor $x$ i.e. unique entry way ID. On each stereo every VAP can be independently allowed or impaired apart from the by default used VAP 0 which is always allowed. VAP 1-15 must be configured to be allowed or disconnected however to impair VAP 0 from the BSSID the stereo itself must be impaired from the BSSID because VAP 0 is actually assigned to the BSSID of the actual AP.

The concept and use of VAP is well learned and needs least explanation as of this article is concerned, based on the knowledge base assumption. However nominal explanation of limitations of VAP would be considered to lay solidity in the use of Multi-channel VAPs. As same channel is shared between all APs if client management is concerned. It means that if one station is moving away from one AP, then, that AP will start searching for neighboring AP to proceeds with the handoff, and for this both AP have to share the same channel on both APs, where probability of interference is quite high. In this article the use of VAP with multi-channel techniques is proposed because it addresses the handoff problem just as it wanted to be in seamless handoff and connectivity disruption. In this technique the APs will search for the neighboring AP using different channel from the one it is using to send beacons to the station.

Fig. 1 shows the handoff procedure of a moving station. This shows when a station decided to get away from an AP and moving closer towards neighboring AP, the handoff procedure will initiated and the station which is originally connected to the AP through a dedicated VAP will communicate with the neighboring AP with the aim of shifting the association state to the new AP. This virtual operation will be proceed with the seamless handoff, as station will be receiving the beacons reception from another channel of the same VAP and the shifting of association will occur through the different channel.

Channel Switch Announcement is the mechanism of sending a message encapsulated in AP beacon by AP to the moving station which lets the station know about its intention to change the channel. Normally station stop receiving data from the AP until it re-associates itself to the new AP. In normal circumstances, the station changes its channel when the channel is too noisy, high on traffic or has greater probability of interference. This CSA is element of 802.11 standards. [3].

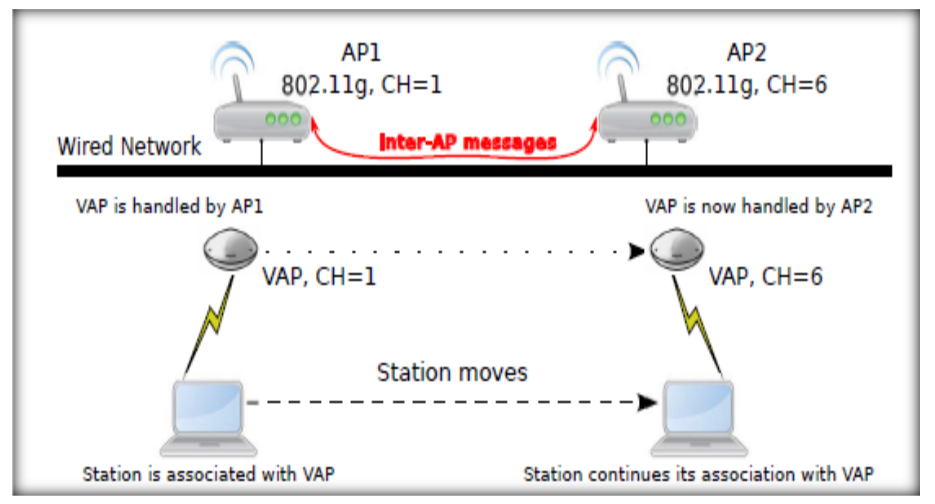

Fig. 1. Mobility Management Handoff and Inter-AP Messages. 
Logically when a station is already associated with an AP and moves out of the range of it, it has to re-authenticate, reassociate to the new AP on same or may be on different channel, so the old AP thus changes its channel to transmit the data to the channel of new AP. During this the station will face a downtime as it will not be receiving any beacon from the older or new AP until the handoff procedure is completed and same will be with the APs.

So having virtual access points working not using multiple channels for the transmission will not solve the problem of latency and downtime in handoff procedure. This is why the multi-channel virtual access points are suggested as discussed earlier. Even after this, if there will be no AP at the new location of the station, the standard handoff procedure will be initiated which will also be true for the legacy of 802.11 WLAN devices.

In this section MVAP protocol is analyzed with great details, the communication between Station and both APs will occur in the following manner as shown in the figure (Fig. 2) below.

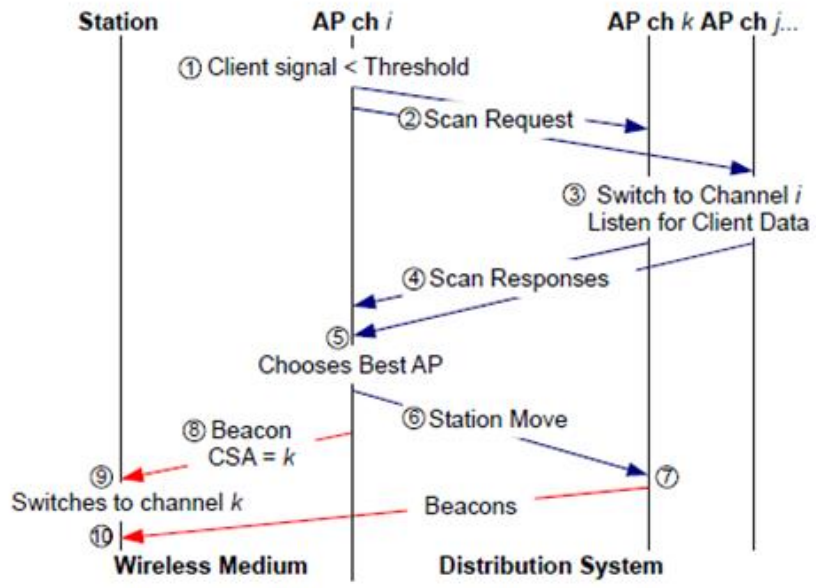

Fig. 2. Multi-Channel VAP.

1) A station (STA) is starts moving from the $\mathrm{AP}_{\mathrm{i}}$ to which it was associated through channel say $\left({ }_{i}\right), A_{i}$ will start recognizing the motion of STA and its dropping signal strength which is decreasing than the threshold.

2) Using Distributed system the $\mathrm{AP}_{i}$ will send a Re-Scan beacon to the neighboring APs, as all the APs have the information about its neighboring APs like MAC address and IP.

3) All APs except (i) will tune themselves to same channel as of AP(i) which is been sent to them by AP(i) in this case channel i. All APs will start listening to the packets receiving from STA.

4) Any AP which will successfully receive beacons from the STA will send a response to Scan Request to AP(i) using the same Distributed System Technique.

5) $\mathrm{AP}(\mathrm{i})$ receives the Scan Responses and analyze them according to the direction of the motion of STA, and will choose best AP with better signal strength than its own.
6) Again using Distributed System, AP(i) will send Station Move message to the chosen $\mathrm{AP}(\mathrm{k})$, as shown in figure.

7) As soon as $\mathrm{AP}(\mathrm{k})$ will receive the Station Move message from AP(i), it will start sending beacons to the station STA.

8) Now AP(i) being nice to the STA will send the beacon of channel switch announcement CSA to switch its channel to $\mathrm{k}$. Thus CSA will force the STA to switch its channel to $\mathrm{k}$ where further communication will take place.

9) STA receives the CSA element from $\mathrm{AP}(\mathrm{i})$ and switch its channel to $\mathrm{k}$.

10)STA changes its AP and channel consequently without losing connectivity.

\section{IMPLEMENTATION}

The implementation of the suggested solution of mVAP will be done using PACMAC i.e. packet manipulation framework tool, designed to capturing and manipulating the 802.11 frames in the network. This tool allows monitoring and injects the manipulated frames for quick prototyping of adaptation or alteration of IEEE 802.11 management and data functions. Maria Eugenia Berezin, Franck Rousseau, Andrzej Duda, updated this tool for the implementation of mVAP scheme in it. This tool is available for download at http://pacmap.ligforge.imag.fr/ (Maria Eugenia Berezin, 2014).

MadWiFi driver was introduced in this tool, which listens to all the packets in monitoring mode, without filtering them, and at the same time MadWifi injects the manipulated frames in the network and sends them over the wireless medium.

Virtual network kernel interface TUN/TAP interface, used for providing packet reception and transmission for user space programs. [4] TUN stands for network tunnel and TAP is the network tap, network layer and link layer devices respectively. Tap network device used to inject or retrieve the frames from the network, while, here PACMAC uses the TAP tool to inject the incoming data packets over WLAN network in to the kernel space from user space and finally in to the kernel network or IP stack for further processing as shown in Fig. 3. While inter-AP messages uses a normal TCP socket for the exchange of packets.

As per this situation, the question of IP change may arises if there is a change in switching the AP because each access point have its own IP leasing mechanism which is updated in the ARP table of the access point, but here all access points are in the same sub-network [5].

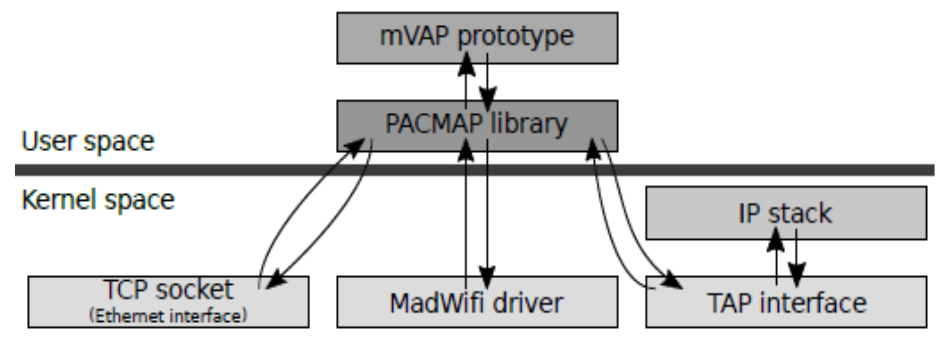

Fig. 3. PACMAP Architecture and M-VAP Implementation. 


\section{A. AP to AP Communication and Inter-AP Protocol}

As far as communication between different devices is concerned not only the network mechanism among them is concerned but also protocols are to be defined. Protocols are the set of instruction, rules and standards which are to be defined that on what conditions the devices must communicate, likewise, FTP, SMTP, X.25 and still growing list. The communication between APs, between same or multi-vendor devices also needs the protocols to be defined in order to make them communicated with each other.

IAPP (inter access point protocol) or $802.11 \mathrm{~F}$ provides wireless access point communication and maybe taken as an optional extension to 802.11. The IEEE 802.11 does not postulate the communication between APs as in case of user's roaming displacement from one AP to another AP. According to the requirement of this research, during handoff period the STA's security is vandalized, thus this protocol specifies the implementation of unique association throughout the BSS and ESS and for the secure exchange of STA from one AP to another [6].

Ethernet wired network normally interconnects access point in current settings likewise Distributed system are responsible for the inter AP communication. TCP is the reliable medium of sending messages between APs and includes the following information:

1) Scan request: Scan request is initiated by the $\mathrm{AP}(\mathrm{i})$ to which station STA is connected with before handoff procedure as shown in Fig. 2 and is sent to the neighboring APs it knows around it. This scan request included all information about the station STA like, MAC address, IP address and station channel currently tuned on, and also BSSID.

2) Scan response: Scan Response is acknowledgement or the response from the neighboring APs in turn of receiving scan request from $\mathrm{AP}(\mathrm{i})$. This response beacon will contains information about the MAC address \& IP address of the station STA, RSSI Received Signal Strength Indicator (to let the AP(i) know whether the signal STA will receive are better than the old AP or below its threshold) and also the channel of itself i.e. channel of the new AP.

3) Station move: when the AP(i) will find out an AP with the better RSSI than itself it will choose the best AP and send the station move beacon to that AP. This beacon will include the information about STA MAC \& IP addresses along with the beacon interval and channel count.

TCP sockets are used between the AP Ethernet interfaces to implement the inter-AP communication. So that each AP listens on the dedicated port and the communication of messages to other APs is carried out through these sockets.

\section{B. Channel Switch Announcement}

As detailed earlier, Channel Switch Announcement is used to by the AP to advertise its channel switching activity [7]. The information included in the CSA beacon transmitted by the AP is as follows:
1) Channel switch count: Associated devices with an AP must know that the AP is switching to another channel and it is mandatory to know that when it will switch, for this purpose before switching to new channel, beacons transmitted by the AP includes beacon counts, which decreases respectively with each transmitted beacon.

2) Channel switch mode: Channel switch mode includes information about the restrictions and limitation on transmission before switching like band selection and interference due to traffic on adjacent channels.

3) New channel number: It must include and advertise information that to which channel number it is switching and the neighboring APs and STAs should re-associate itself to this new channel now.

The channel switch announcement element is formatted is as follows in Fig. 4.

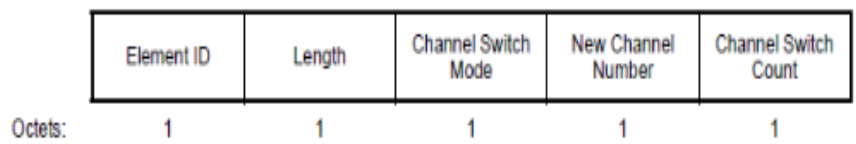

Fig. 4. Beacon Contains CSA Information.

The discussion above is to establish the fact that the each station STA is associated with its own Virtual Access Point and receives custom beacons from the AP and not the normal or universal one which are being transmitted to other APs of course. This element will be the part of beacon only after the new AP is chosen. According to the scenario of this research the AP will transmit 3 consecutive beacons, with the decrement of each transmitted beacon in the CSA count. The interval between the transmitted beacons will be $100 \mathrm{~ms}$. When the value after 3 beacons will reach to 0 to $300 \mathrm{~ms}$ the AP will stop transmitting beacons to the station.

\section{EVALUATION AND RESUltS}

The scenario chosen to carry out the experiment, three laptops and one desktop is configured to work as per the requirement of the experiment. Two laptops are configured to work as Access Points, however, real access points can also be used in place of laptops, but the results from each node are required so laptops are configured as AP1 and AP2. The third laptop is configured as station STA which is mobile and will move away from one AP towards the other AP to let it undergo handoff process. The desktop computer on a wired network is used to provide internet access to the laptops which are being used as Aps.

Fig. 5 demonstrated the movement of the client (mobile station STA) from AP1 to AP2 this is clear that the STA was first associated to AP1 on channel 1 and then starts moving towards AP2 where it will be receiving beacon on channel 6 which will now become its new default AP. As shown in the diagram it flows back to near AP1 where it is not in range of AP2 and then associated itself back to AP1. 


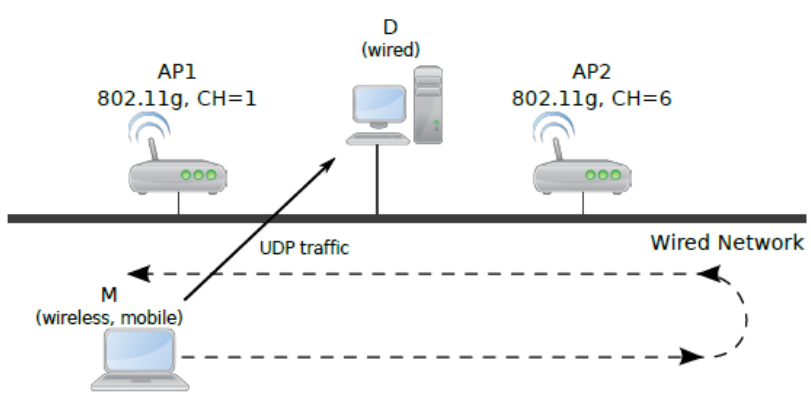

Fig. 5. Experimental Setup.

During all this transition from AP1 to AP2 and then back to AP1, the performance of the handoff can be monitored on Desktop terminal D, where we will receive the UDP from the station. For the sake of experiment, we will consider the packets of VoIP; these packets will be sent in continuous stream of UDP packets not be actual but mimicry of the original VoIP packets and are generated by different audio codecs like G729 \& G.728. Voice codecs used for the evaluation in this research are as in Table I.

TABLE I. VOICE CODECS AND BEACON INTERVAL USED IN EVALUATION

\begin{tabular}{|r|c|c|c|}
\hline \multicolumn{1}{|l|}{ Codec } & Bit Rate(Kbps) & $\begin{array}{l}\text { Voice Payload } \\
\text { Size (Bytes) }\end{array}$ & Interval (ms) \\
\hline G.728 & $8 \mathrm{kbps}$ & 20 & 20 \\
\hline G.729 & $16 \mathrm{kbps}$ & 60 & 30 \\
\hline G.711 & $64 \mathrm{kbps}$ & 160 & 20 \\
\hline
\end{tabular}

IPref tool is used for the generating the traffic of UDP packets, IPref tool is a network tool for monitoring and tuning. This tool can be used in cross platform tool that can produce standardize performance measurements for very network. The final frame of the UDP packet contains actual payload of audio codec plus 12 bytes reserved for the Real Time Protocol (RTP) as a header.

\section{A. Performance Analysis}

The performance in handoffs directly influenced by the latency of the packets arrived when the STA switches the AP and channel. So the time taken between the packet arrivals will be the latency due to handoff procedure. Such analysis is called packet inter-arrival Time or IAT. In terms to equation, this will be denoted by the nth packet and preceding packet n-1.

$\mathrm{IAT}=\operatorname{IAT}(\mathrm{n})-\operatorname{IAT}(\mathrm{n}-1)$

As shown in Table I, the inter arrival time of the packets is not more than $30 \mathrm{~ms}$ which justifies the threshold of maximum $150 \mathrm{~ms}$ of delay which is been thought complementary for the VoIP communication. It is also been noticed that only packet is lost during handoff process due to ARP table actualization. Consequently the transition from AP1 to AP2 or back to AP1 shows no interruption in the VoIP communication. A same situation can be observed in the CDF (cumulative Distribution Function of inter-Arrival Time (IAT) of the UDP packets in the Fig. $6(a, b, c)$ below, there are three different results for three different audio codec i.e. G.729, G.728 and G.711, respectively.

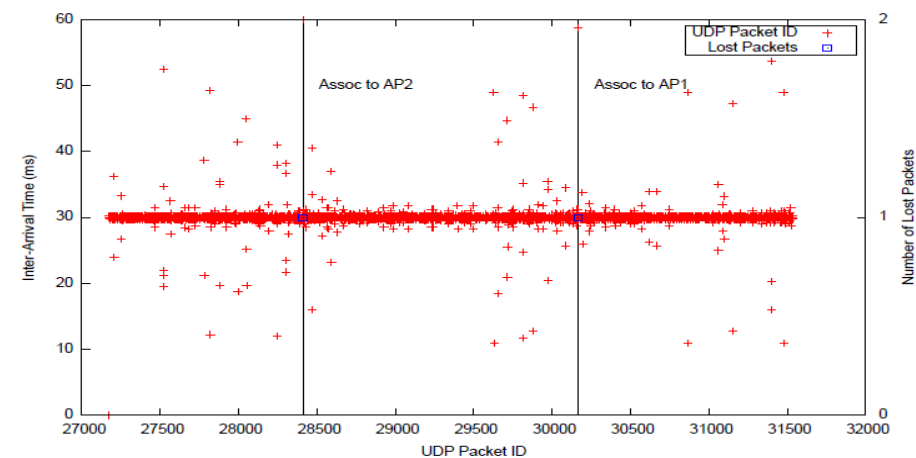

(a). G.728 Codec

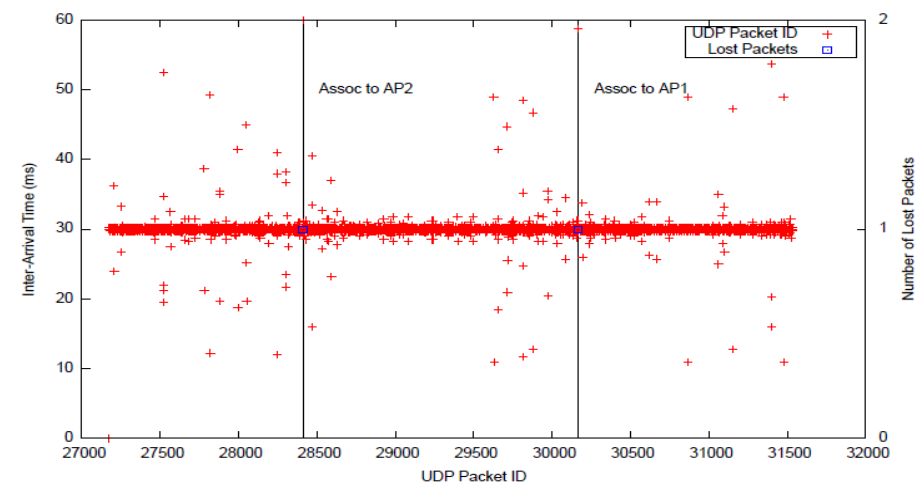

(b). G.729 Codec

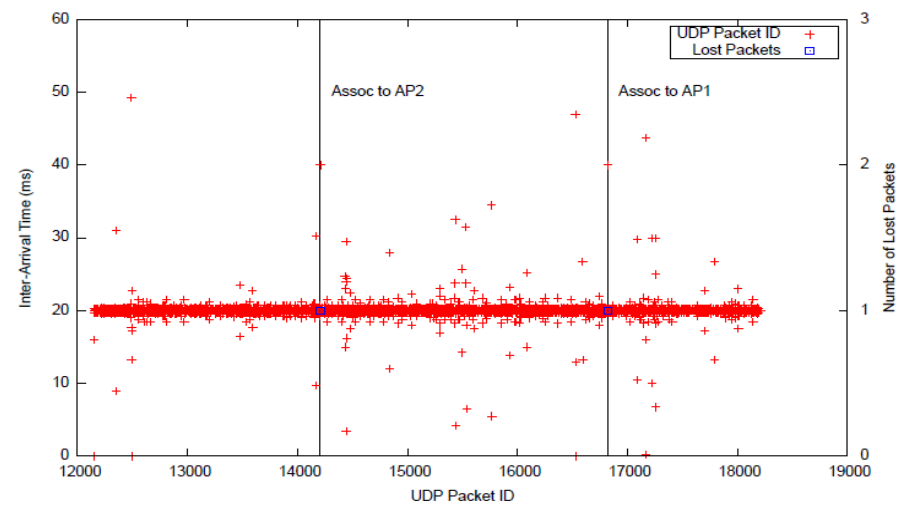

Fig. 6. (c) G.711 Codec.

It can be observed that none of the value of latency in the IAT passes the $20 \mathrm{~ms}, 30 \mathrm{~ms}$ and $20 \mathrm{~ms}$ respectively. Statistical analysis will show its, mean, $90^{\text {th }}$ percentile and standard deviation of the noted readings is shown in Table II.

The results in Table II shows the hypothesis developed in the research that multi-channel virtual access points can manage and handle the handoff procedure without interruption in the connectivity of the station to the transmitting APs.

TABLE II. IAT DISTRIBUTION FOR EACH CODEC USED

\begin{tabular}{|l|l|l|l|}
\hline Codec & Mean & $\mathbf{9 0}^{\text {th }}$ percentile & Std. Deviation \\
\hline G.728 & 0.02001 & 0.02024 & 0.00072 \\
\hline G.729 & 0.03001 & 0.03023 & 0.00163 \\
\hline G.711 & 0.02001 & 0.02024 & 0.00076 \\
\hline
\end{tabular}




\section{DISCUSSION AND FUTURE WORK}

As in this research the solution purposed to use multichannel virtual access points instead of virtual access points, the proposed solution emphasize on switching the channel while switching the AP. However, it can also be done in a different way that may include some different protocols and techniques. One such solution can be switching the AP without changing the channel, which will produce more reliable results and the IAT can be even better. This can also be done by introducing another wireless card which will be always listening to the packets receiving from the moving station. With new wireless card AP will be able to exchange packets with its client without any interruption while it is scanning for the AP.

The approach used in the solution proposed in this research is based on the better signal strength and reception which is being received by the station at that very instant i.e. instantaneous signal strength, which can arise issue in case of any emergent change of direction of the moving station. For this to overcome, complete analysis of the most likely movement of the station can be helpful for performing handoff. For this Displacement Vector \& Pointing Vector from Station to AP it is connected to and the next possible AP to be connected can give somehow deep information such as: historical information and trends about the movement of the station [8].

Finally, a recommendation on the security issues is mandatory at this stage. Authentication process must be included for the new AP, because in most the WLAN technologies, security is the major concerns in the current deployments [9]. The suggestion for the researchers is to ponder on this aspect of security, specifically 802.11i authentication protocol.

\section{CONCLUSION}

In this research paper a solution has been presented which emphasizes on the use of multi-channel virtual access points for the 802.11 WLANs. In result of this latency of the handoff procedure is decreased to carry on continuous communication between AP and mobile station. The advantage of low latency will influence in expedite communication especially in stations using multi-media applications or VoIP communications. This solution is also supported with the inter-AP protocol to send Scan Request to the neighboring AP to let them know about the movement of the station.

Other technicalities to carry out real world experiment included PACMAP framework to monitor and analysis the transmitted beacons and mimicking the VoIP UDP packets. The wireless card used in this experiment was Atheros based chipset, and the PACMAC was supported by the MadWifi driver, in user's space. As shown, three types of codecs used for the experiment, and results shows that the IAT doesn't cross the threshold of $150 \mathrm{~ms}$ while performing handoff, hence, creating no disruption in the communication.

Concluding arguments included the suggestion of multiradio environment, adding security features and suggesting a mechanism to trigger the scanning of the neighboring AP and various election routines of the APs. Our next research will be focusing these points, as well as will include vertical handoff techniques and impact of handoffs on the performance of 802.11 in WLANs to build full-bodied solution.

\section{REFERENCES}

[1] Mishra, M. S. a. W. A., 2003. An Empirical Analysis of the IEEE 802.11 MAC Layer Handoff Process. SIGCOMM Comput. Commun, 33(2), pp. 93-102

[2] Kim, H.-S., Park, S.-H., Park, C.-S., Kim, J.-W. and Ko, S.-J., (2014). Selective Channel Scanning for Fast Handoff in Wireless LAN using Neighbor Graph. Proceedings of ITC-CSCC, Sendai/Matsusima. (), pp.303-316

[3] Developer Network, 2013. http://msdn.microsoft.com/en us/ library /aa292197(v=vs.71).aspx. [Online] Available at: http://msdn.microsoft .com/enus/library/aa292197(v=vs.71).aspx

[4] Anon., 2013. Configuring Virtual Access Points, s.l.: s.n.

[5] ITU, (2003). International Telephone Connections and International Telephone Circuits. International Telecommunication Union-TG 114.

[6] Mhatre and K. Papagiannaki, "Using smart triggers for improved user performance in 802.11 wireless networks," in MobiSys, 2016.

[7] Y. Liao and L. Gao, "Practical Schemes for Smooth MAC Layer Handoff in 802.11 Wireless Networks," in WoWMoM, 2016.

[8] Raghavendra, Ramya, Elizabeth M. Belding, Konstantina Papagiannaki and Kevin C. Almeroth, (2017). Understanding Handoffs in Large IEEE 802.11 Wireless Networks. IMC'07, October 24-26, 2017, San Diego, California, USA.

[9] H. Velayos and G. Karlsson,"Techniques to reduce the ieee $802.11 \mathrm{~b}$ handoff time," in IEEE ICC, 2014. 